\title{
BASIC DATA
}

\author{
A. ACKER \\ Observatoire Astronomique de Strasbourg \\ 11 rue de l'Université 67000 Strasbourg, France
}

\section{Introduction}

Basic data on planetary nebulae concern all the fields related to these objects. As an illustration of the increasing importance of planetary nebulae in large fields of contemporary science, let me note that, in a communication for a conference on "bibliometrics, informetrics and scientometrics", Bassecoulard and Zitt (1994) chose planetary nebulae as the best example of a complicated network allowing and needing interesting cluster analysis.

A steadily increase of papers devoted to planetary nebulae is observed since the publication of the famous Catalogue of galactic planetary nebulae by Perek \& Kohoutek in 1967. The Centre de Données astronomiques de Strasbourg (CDS, founded in 1972 and extended to non-stellar objects in 1983) collects, homogenizes, distributes, preserves astronomical information, through several services. Until 1987, the 'star' of the data base SIMBAD was NGC 7027 with a total of about 760 references (see Acker, 1989). Now NGC 7027 remains in the leading-group with 1191 references but at the 7th position, the first positions being occupied by galaxies (M31 no. 1 with 1660 group references, LMC with 1650 references) and SNR (SN87A: 1588 ref.; M1: 1234 ref.). The next PN in the SIMBAD "hit parade" are IC 418 (693 ref.), NGC 6543 ( 585 ref), BD $+30^{\circ} 3639$ (535 ref.).

Fig. 1 shows the evolution since 1983 of the numbers of articles referenced in SIMBAD, with a title containing the term "planetary nebula" (including the BAAS reports, about 10 per year). Two peaks correspond to specific european workshops (1987: Planetary and Proto-Planetary nebulae: From IRAS to ISO, Vulcano, ed. Preite-Martinez; 1993:Nuclei of Planetary nebulae: Observations versus theory, Bachotek, ed. Tylenda, Acta Astronomica 43). The Proceedings of the Workshop Planetary nebulae with Wolf-Rayet type nuclei hold in Ven, Sweden, August 1995, are published 


\section{BASIC DATA}

Figure 1. Evolution of the papers devoted to the planetary nebulae

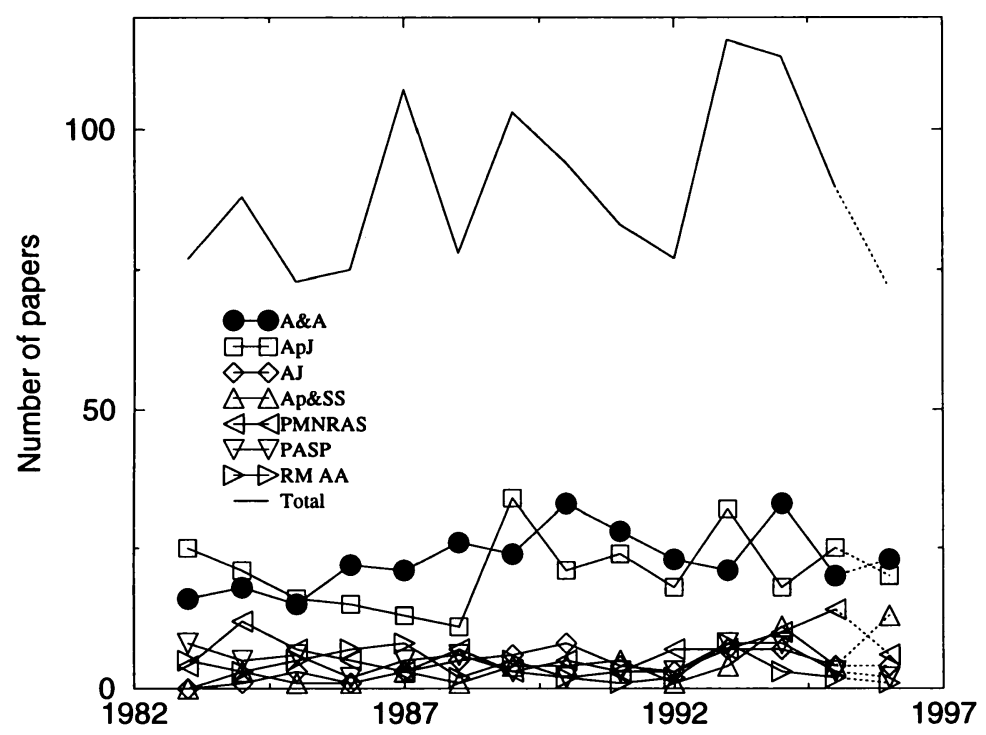

in Astroph. Space Sc, 238/1, eds. Lundström \& Stenholm (Kluwer, 1996). The Proceedings of the Colloquium Asymmetrical Planetary Nebulae (Annales of the Israël Physical Society 11, eds . Harpaz \& Soker, 1995), and the Proceedings of the IAU symposia 103,131, 155 devoted to planetary nebulae are not included in Fig. 1.

Since 1995, abstracts of recently accepted papers and various information (dissertations, meetings, jobs,...) are available through the $A G B$ Newsletter, an excellent electronic monthly publication dedicated to stellar evolution on the AGB and beyond (Eds. T. Forveille and C. Kahane; agbnews@gag.observ-gr.fr).

2. The catalogues of galactic planetary nebulae and the number of known planetary nebulae

The total number of PN in our Galaxy and their distribution is still uncertain, limiting our knowledge of the death rate of low mass stars, and of the rate of enrichment of the interstellar medium, that constrain the chemical evolution of the Galaxy. 


\section{A. Acker}

\subsection{THE CATALOGUES}

The first Catalogue of galactic planetary nebulae (PKCGPN, Perek \& Kohoutek, 1967) contains 1063 objects. The Strasbourg-ESO catalogue of galactic planetary nebulae (SECGPN, Acker et al, 1992) provides information of 1143 true or probable PNe (846 coming from the PKCGPN and 218 from supplementary lists compiled by Kohoutek), and listed 347 possible PN with an unclear status, 330 objects being rejected from the community of planetary nebulae. This selection was mainly based on spectrometric observations performed by Stenholm and Acker for all suspected PN (about 1500 objects have been inspected, using the criteria presented in the introduction of the SECGPN).

Note that the data from SECGPN are yet not included in SIMBAD (which give the names and data of $\mathrm{PNe}$ as appearing in the literature). The SECGPN is directly available on the CDS Anonymous ftp account on node: cdsarc.u-strasbg.fr (130.79.128.5) in the directory /pub/cats/V/84, or on the WWW address: http://vizier.u-strasbg.fr/cgi-bin/wcbCatSelectPg?GPN

\subsection{NEW PLANETARY NEBULAE}

The brightest "bona fide" $\mathrm{PNe}$ are already known. Objects newly discovered are either classic PNe but very faint and difficult to find (generally they are extended and old), or PN-like objects discovered through the typical signature from the nebular plasma or the dust in the "invisible" domain.

Therefore, the newly discovered $\mathrm{PNe}$ result essentially from four different surveys.

- The first sample is defined by using the classical search of nebulosity done by the Innsbruck's group on photographic plates. With the advent of CCD detectors, Napiwotzki et al. found very large and faint nebulae around hot subdwarfs and sdO stars,

- The 2nd method, already used by Sabbadin in 1986, is based on a comparison of red and infrared POSS plates (Cappellaro et al, 1994; Zanin et al, this volume). - The third sample concerns objects with specific IRAS colors (see Pottasch et al, 1988). About 130 IRAS objects were identified as being PNe through the detection of $6 \mathrm{~cm}$ emission (indicating the presence of ionized gas). The PN nature is confirmed for some objects by optical spectroscopy (Van de Steene et al, 1996), or through near-infrared photometry (Garcia-Lario et al, 1996).

- The 4th group results from systematic surveys in specific galactic populations. 133 of the 151 globular clusters are surveyed by Jacoby \& Fullton (1994). Of the 12 PNe expected, two new PNe are found, in NGC 6441 and Pal 6. On the other hand, the galactic halo population was recently enriched by PN found around high latitude blue objects (LSIV- $12^{\circ} 111, \mathrm{BD}+33^{\circ} 2642$ ).

-Deep surveys of the galactic bulge were conducted recently by Beaulieu (1996), Kohoutek (1994), Jacoby and Van de Steene (1996-1997, in preparation), increasing by about 120 objects the galactic bulge planetary nebulae population.

The first Supplement to the Strasbourg-ESO Catalogue of galactic Plan- 


\section{BASIC DATA}

etary Nebulae (Acker et al, 1996) includes 243 true or probable PN (with the finding charts) and 142 "possible" PNe. An additional list of $56 \mathrm{PNe}$ are newly discovered by Zanin et al (this issue).

\subsection{NUMBER OF KNOWN PLANETARY NEBULAE}

The number of known galactic planetary nebulae is steadily increasing (see Table 1), and may include in the future a significant part of the about 3000 unidentified IRAS sources with PN colours. However the size of the observable sample should remain far from the total number of PN expected in the Galaxy (7 000 to 40000 ). The incompleteness is caused by (i) the interstellar extinction hiding PN near the galactic plane, (ii) the large distances (the nearest PN, S 216 and NGC 7293, are situated at about 200 pc), and (iii) the fact that it is progressively difficult to find PN as they become older (see Pottasch, 1996); in particular, an evolved PN poor in dust will not have been detected by IRAS.

TABLE 1. Number of known planetary nebulae

\begin{tabular}{|c|c|c|c|}
\hline Year & objects called PN & true - probable PN & possible PN \\
\hline 1967 PKCGPN & 1063 & $(846)$ & \\
1992 SECGPN & 1820 & 1143 & 347 \\
1996 1st suppl. & +385 & +243 & +142 \\
\hline
\end{tabular}

\section{Main data}

\subsection{COORDINATES}

- Through precise astrometric measurements of PNe from the Magellanic Clouds, Leisy (1996) and Leisy et al (1996) pointed out that the published positions are very uncertain.

- Comparison on Guide Star Catalogue (GSC) positions and coordinates reported in the SECGPN done by Brian Skiff (1995) for the brightest PNe shows that the reported position is accurate in only one-third of the cases. Good recent positions are given by Kohoutek (1986). In some cases, the PNe appear in contemporary astrometric catalogues: the best position is from PPM or ACRS (see Acker, 1997). 


\section{A. Acker}

\subsection{VELOCITIES}

- In 1992, a radial velocity was reported in the SECGPN for 577 PNe. Beaulieu (1996) gives the radial velocity for 370 galactic bulge PNe. Durand et al (1996) present an up-to-date compilation of the velocity of 856 galactic $\mathrm{PN}$, calibrated through new high-resolution velocities on $119 \mathrm{PNe}$ mostly located in the galactic bulge (Zijlstra et al, 1996).

- Expansion velocities are studied through high resolution observations by Acker \& Zijlstra (120 GBPN, in preparation) and by Tamura et al (78 PN, this volume).

\subsection{MONOCHROMATIC IMAGES AND DIAMETERS}

- A rich variety of colorful or deep images of PNe are offered in recent years, the most spectacular being those taken at high spatial resolution by the HST. On-line images are distributed by C.Y. Zhang, B. Balick, G. Jacoby. The IAC (Instituto de Astrofisica de Canaria) group undertook a homogeneous morphological survey of northern galactic PN (Manchado et al, $1995 ; 1996$ this volume). An imaging and spectroscopic survey of the Abell PN is undertaken by Walton \& Walsh (this volume).

- Diameters are an important parameter in particular for determining the distances and the masses of PNe (see Beding \& Zijlstra, 1994). The observed distribution results from the combination of the evolution of the nebula and of their distance: compact young nebulae looking stellar and bright are detectable at large distances.

\subsection{FLUXES}

- Visible - Nebular line intensities are presented by Cahn et al (1992), Perinotto et al (1994), Costa et al (1993), Kingsburgh \& Barlow (1994), Cuisinier et al (1996) and Exter \& Barlow (1996). A catalogue of HeII 4686 line intensities in galactic PN is performed by Tylenda et al (1994). The intensities of selected lines from the spectrometric survey at low resolution of about $900 \mathrm{PNe}$ by Stenholm \& Acker are published in the SECGPN (book and on-line). The full spectra will be accessible by WWW in a near future. A "Catalogue of relative emission line intensities observed in PN" produced by J.B. Kaler \& L. Browning (Univ. of Illinois), and R.A. Shaw (Space Telescope Science Institute) is available in a digitized version. Absolute spectrophotometric observations were made by Dopita and Hua (1996, this volume) for some 50 bright southern $\mathrm{PNe}$, in order to provide "standard" fluxes allowing future calibration. General studies of central stars of $P N$ are concentrated on emission-line nuclei (see Tylenda et al, 1993; the papers of the Ven Proceedings, 1996; and the review of Hamann, this issue). 


\section{BASIC DATA}

- $U V$ and X-ray - Quigley et al (1993) performed a high dispersion IUE atlas of central stars of PN. Fruscione et al $(1993,1995)$ report on EUVE observations. Feibelman (1994) discusses X-ray PNe. Hoare et al (1995) reported on ROSAT observations. The release of the ROSAT All-Sky Survey Bright Source Catalogue is anounced by J. Engelhauser in an IAU Circular (1996). Diffuse all-sky maps derived from the RASS will be put to the WWW (http://www.rosat.mpe-garching.mpg.de/survey/sxrb/).

- IR - A near infrared ( $\mathrm{J}, \mathrm{H}, \mathrm{K}, \mathrm{L}$, , and $\mathrm{M})$ survey of northern $\mathrm{PNe}$ is conducted by the IAC group in collaboration with astronomers from Mexico and Groningen. Philips \& Cuesta (1994) observed 77 evolved PNe (in complement to the earlier Persi et al study of 117 compact $\mathrm{PNe}$ and related objects). In the framework of detection of new PNe and PPNe among about 1080 IRAS sources with colours like PN, García-Lario et al (1996) present NIR photometry of a new sample of 225 sources (113 classified as PN).

A survey in the near-infrared and narrowband images is completed by Latter et al (1995), Hora et al (1996, this volume). Meixner et al (1994) conducted mid-IR imaging of carbon rich proto-PNe. Kastner et al (1996) conducted an imaging survey of $\mathrm{H} 2$ emission (survey available via anon.fp, node space.mit.edu, directory $\mathrm{ftp} / \mathrm{pub} / \mathrm{jhk} /$ planetaries).

- Radio - Main radio surveys were conducted with the VLA in the 90's by Zijlstra et al., and by Aaquist \& Kwok. Ratag \& Pottasch (1991). Van de Steene \& Pottasch $(1993,1995)$ reported on radio continuum $(6 \mathrm{~cm})$ measurements of IRAS sources, about 100 in the galactic bulge, 90 in the southern sky, and 80 in the northern sky. Kwok \& Aaquist (1993). Pottasch \& Zijlstra (1994) reported on VLA measurements. Hu et al $(1993,1994)$ conducted a systematic study of IRAS selected proto-PN candidates $(\mathrm{OH}$ and $\mathrm{CO}$ observations). Millimetre and submillimetre continuum observations of PN were done by Hoare et al (1992). Ott et al (1994) presented an up-dated list of radio flux $(0.7$ to $21 \mathrm{~cm})$ calibrators.

As a concluding remark, let me return to the generic name "planetary nebula". This term "is misleading, the objects of this kind have nothing to do with planets at all" (Stenholm, 1993). It is pleasant to note that Soker (1996) argues that properties of elliptical PN hint that giant planets commonly exist near main sequence stars, and therefore planetary nebulae can tell us about planets!

\section{Acknowledgments}

Many thanks to François Ochsenbein (CDS), Bryan Skiff, Monique Hamm (Library), Olivier Fliegans and Nicolas Lehner. Financial support was pro- 


\section{A. Acker}

vided by the GDR 968 "Milieux circumstellaires".

\section{References}

Acker A., 1997, Bulletin du CDS, in preparation

Acker A., 1989, IAU Sympos. 103, S. Torres-Peimbert ed., 39

Acker A., Ochsenbein F., et al, 1992, SECGPN, ESO publication

Acker A., Marcout J., Ochsenbein F., et al, 1996, First supplement to the SECGPN, Public. Observatoire de Strasbourg.

Bassecoulard E., Zitt M. , 1994 : "Fourth international conference on bibliometrics, informetrics and scientometrics", Berlin, Sept. 1993, Public. OST (Obs. Sciences et Techniques, rue de Vaugirard, 75006 Paris, France)

Beaulieu S., 1996, PhD thesis, Australian National University

Bedding T.R., Zijlstra A.A., 1994, $A$ \& $A, \mathbf{2 8 3}, 955$

Cahn J.H., Kaler J.B., Stanghellini L., 1992, A \& A Suppl. Ser., 94, 399

Cappellaro E., Benetti S., Sabbadin F., Salvadori L.,et al, 1994,MNRAS, 267, 871

Costa R.D.D., de Freitas Pacheco J.A., Maciel W.J., 1993, $A$ \& $A$, 276, 184

Cuisinier F., Acker A., Köppen J., 1996, $A$ \& $A$, 307, 215

Durand S., Acker A., Zijlstra A., et al, 1996, $A$ \& $A$ submitted

Exter K., Barlow M., 1996, private communication

Feibelman W.A., 1994, PASP, 106, 756

Fruscione A., Abbott M., Drake J.J., 1993, BAAS, 25, 1371

Fruscione A., Drake J.J., McDonald K., Malina R.F., 1995, ApJ, 441, 726

Garcia-Lario P., Manchado A., Pych W., Pottasch S.R., 1996, A \& A, in press

Gutierrez-Moreno A., Moreno H., Cortes G., 1995, PASP, 107, 462

Hoare M., Roche P.F., Clegg R.E.S., Hill P.W., 1992, MNRAS, 258, 257

Hoare M.G., Barstow M.A., Werner K., Fleming T.A., 1995, MNRAS, 273, 812

Hu J.Y., Slijkuis S., De Jong T., Jiang B.W., 1993, A \& A Suppl. Ser., 100, 413

Hu J.Y., Te Lintel Hekkert P., Slijkuis S., et al, 1994, A \& A Suppl. Ser., 103, 301

Jacoby G., Fullton, 1994, BAAS 26, 1384; 1996, ApJS in preparation

Kastner J.H., Weintraub D.A., Gatley I., Merrill K.M., Probst R.G., 1996, ApJ, in press

Kingsburgh R.L., Barlow N.J., 1994, MNRAS, 271, 257

Kohoutek L., 1986, $A$ \& $A, \mathbf{1 6 2}, 232$

Kohoutek L., 1994b, Astron. Nachr., 315, 3, 235

Kwok S., Aaquist O.B., 1993, PASP, 105, 694

Latter W.B., Kelly D.M., Hora J.L., Deutsch L.K., 1995, ApJ Suppl. Ser., 100, 159

Leisy P., 1996, PhD thesis, Observatoire de Paris

Leisy P., Dennefeld M. et al, 1996, Astron.Astrophys. Suppl. Ser. in press

Manchado A., Guerrero M.A., Stanghellini L., 1995, BAAS, 27, 865

Meixner M., Graham J.R., Skinner C.J., et al, 1994, Experimental Astron., 3, 53

Ott M., Witzel A. et al, 1994, $A$ \& $A, \mathbf{2 8 4}, 257$

Perek L., Kohoutek L., 1967, PKCGPN, CSSR press

Perinotto M., Purgathofer A., Pasquali A.,et al, 1994, A \& A Suppl. Ser., 107, 481

Phillips J.P., Cuesta L., 1994, $A$ \& $A S$, 104, 169

Pottasch S. R., 1996, $A$ \& $A, \mathbf{3 0 7}, 561$

Pottasch S.R., Bignell C., Olling R., Zijlstra A.A., 1988, $A$ \& $A, \mathbf{2 0 5}, 248$

Pottasch S. R., Zijlstra A.,A., 1994, $A$ \& $A, \mathbf{2 8 9}, 261$ 


\section{BASIC DATA}

Quigley M.F., Bruhweiler F.C., Feibelman W., 1993, BAAS, 25, 1368

Ratag M., Pottasch S.R., 1991, A \& A Suppl. Ser., 91, 481

Skiff B., 1995, private communication

Soker N., 1996, $A p J$, 460, L53

Stenholm B., 1993, IAU Symp. 155, Weinberger \& Acker ed., 11

Tylenda R., Stasinska G., Acker A., Stenholm B., 1994, A \&A Suppl. Ser., 106, 559

Van de Steene G.C.M., Pottasch S.R., 1993, $A \&$ \& $A$, 274, 895

Van de Steene G.C.M., Pottasch S.R., 1995, $A \& A$, 299, 238

Zijlstra A., Acker A., Walsh J., Stenholm B., 1996, $A$ \& $A$,submitted 